\title{
Interactive Visual Alignment of Medieval Text Versions
}

\author{
Stefan Jänicke* \\ Image and Signal Processing Group, Institute for Computer Science, Leipzig University, Germany \\ David Joseph Wrisley \\ Digital Humanities, New York University Abu Dhabi, United Arab Emirates
}

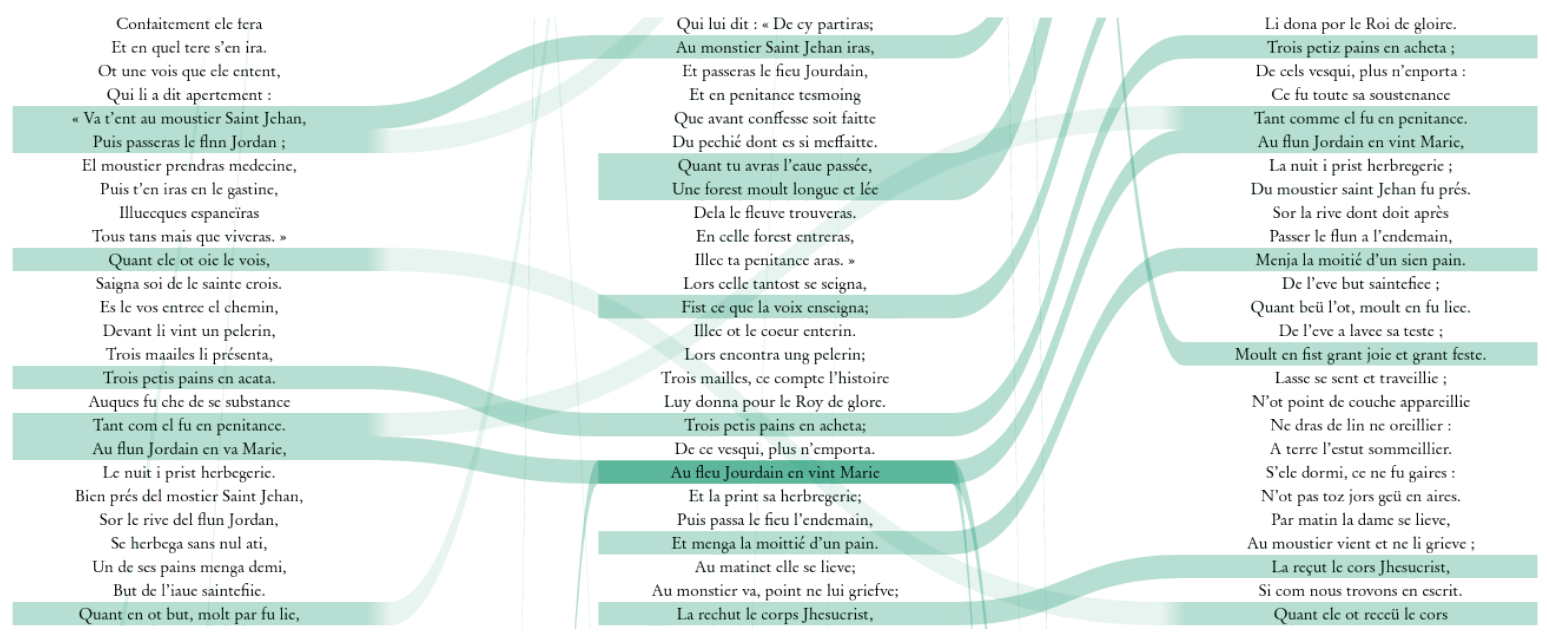

Figure 1: Meso reading of three versions of La vie de sainte Marie l'Egyptienne.

\begin{abstract}
Textual criticism consists of the identification and analysis of variant readings among different versions of a text. Being a relatively simple task for modern languages, the collation of medieval text traditions ranges from the complex to the virtually impossible depending on the degree of instability of textual transmission. We present a visual analytics environment that supports computationally aligning such complex textual differences typical of orally inflected medieval poetry. For the purpose of analyzing alignment, we provide interactive visualizations for different text hierarchy levels, specifically, a meso reading view to support investigating repetition and variance at the line level across text segments. In addition to outlining important aspects of our interdisciplinary collaboration, we emphasize the utility of the proposed system by various usage scenarios in medieval French literature.
\end{abstract}

\section{INTRODUCTION}

In the pre-print age, poetry was recited and passed along orally, but it was also written down and recopied by hand. Textual criticism is the field of literary scholarship that addresses these problems of competing versions of a work. One of the main concerns of the medieval textual scholar is studying different versions of poetic narratives that changed as they circulated and suggesting what such changes can tell us about the creative process. Critics debate what an original written prototype for these similar documents might have looked like, if such a prototype is lost or if it even existed. In this period there are rarely any authorial names associated with texts and material evidence to understand textual transmission is scarce.

*e-mail: stjaenicke@informatik.uni-leipzig.de

†e-mail: djw12@nyu.edu
A textual critic analyzing the tradition of a given work compiles a list of variant readings in related text segments among the different versions-this process is generally called collation. A well-known example of the multi-text situation in literature is that of Shakespeare's plays, first written down by others and subsequently produced in different printed copies. Whereas the identification of variants in the Shakespearean scenario is realtively straightforward due to the predictable breakdown of plays into acts, scenes and speeches by various characters, with certain kinds of medieval texts the variance is much more complex involving reorganization of narrative, interpolation and excision of entire passages making text comparison a non-trivial task for scholars.

In some kinds of medieval poetry, similarities across versions far outweigh their differences, such as the famous medieval French romances of Chrétien de Troyes. There are other text traditions like the medieval French epic which have a high degree of structural variance. Each version shares familiar plot lines, as well as repeated formulae and text segments although not in the same order. This has led scholars to speak of the epic, known as chansons de geste, in medieval French as an admixture of oral performance and written text. It goes without saying that manual collation of romance is significantly easier than of chansons de geste. Automation of this process is rendered particularly difficult by the fact that in medieval French has a highly variable orthography.

One of the best known examples of medieval French literature is the Chanson de Roland, preserved in seven versions, "each of which is a distinct 'version' by virtue not only of its particular wording but also of the selection of other works with which it was copied or bound, its date, provenance, and dialect" [19]. Although Mortier, the first editor of the collected versions of the Chanson de Roland, attempted to create a rough, numerical indexing system for the stanzas across the versions that correspond thematically, in general, for long texts such as the Chanson de Roland, comparing the versions at the line level is next to impossible without computational assistance. The problem with existing, generic systems for visualizing textual 
versions is that they are designed for texts that are mostly similar like Chrétien de Troyes' romance. The textual record provides us, however, with many different, complex textual scenarios.

In order to fill this gap, the authors of this paper-a computer scientist and a medieval literary historian - came together and collaborated on the development of a visual analytics system that supports the task of exploring the textual complexity of medieval orallyinflected poetry. The potential importance of such a system was discussed in our paper at the annual digital humanities conference 2016 [37]. To begin with, we developed a series of prototypes for computation alignment of highly variant text versions, and worked out best practices for visually conveying this variance. The gained insights ensured that the visual analytics system proposed in the present paper provides the medievalist with a full set of functions to explore textual variance in depth on a number of different genres. In summary, our contributions to the visualization community are:

- A visual analytics text alignment approach: In addition to a novel, parameter-driven approach to align highly unstable text versions, we outline a visual analytics process that supports the literary scholar in tweaking parameters for iterative improvement of the computational alignment while keeping its changes visually traceable.

- Multi-level alignment visualization: We present different visualizations that allow for analyzing textual alignments on different text hierarchy levels (whole text, lines, words). In particular, we address the problem of a multi-level visualization of textual transposition [32].

- Meso reading: Beyond the polarization of close and distant reading visualizations, we introduce an intermediate perspective for texts exhibiting complex variance. Meso reading allows to read texts in parallel, while the interdependencies among text versions beyond the level of the line are shown.

We emphasize the utility of our visual analytics alignment system for the complex textual record of the sort that medievalists study, by outlining a set of usage scenarios across various genres in which a line to line correspondence of similarly structured texts is not the organizing principle of the texts. In a storytelling style, we show how each textual scenario exemplifies how the proposed system supports a multifaceted visual analysis of computationally determined alignments, how it can be used to create a persistent comparison of text versions and how it allows the literary scholar to characterize the style of variation of a given textual tradition according to the visual patterns that $\mathrm{s} / \mathrm{he}$ finds in it. In addition, we report experiences of our interdisciplinary work, including evaluative aspects and limitations due to the nature of humanities data.

\section{Related Work}

Our work, which takes its inspiration from medieval literary history and a deep understanding of problems related to the textual transmission of sources, is situated in the domain of the digital humanities. One of the more important aspects of the digital humanities is to support research questions on texts or objects of the historical human record with computational means and to visualize the results. An exhaustive overview of visualization techniques to support the close and distant reading of text is given in a recently published survey paper [31]. None of these techniques allow us fully to visualize the complexity of medieval textual variance. In the following, we take a look at text visualization methods most related to our work.

\subsection{Text Visualizations for the Digital Humanities}

Text as data type has been the basis for numerous innovative visualizations, an overview being given by Kucher et al. [44]. Some text visualization techniques have been designed for, or are applicable to, digital humanities research scenarios. The focus can be a specific literary text or an entire text collection, where visualization provides an abstract view conveying characteristic features of the given textual data. An example for single text analysis might be fingerprint matrices [39] that illustrate the behavior of certain text feature values on various hierarchy levels of the text. The fingerprinting approach can be further used to highlight semantic text features such as changing relationships between characters in a text [49]. In order to serve corpus-linguistic research, the Text Variation Explorer provides various text statistical views to support visually exploration of the most interesting fragment size (analysis window) for a given text [55]. The VarifocalReader also provides different visual interfaces as views onto different hierarchy levels of a given text [43]—pictograms to highlight occurring text patterns, tag cloud summaries for text sections, and two close reading views (digital version and scan). Machine learning techniques and search mechanisms are applied to extract entities, concepts and other artifacts from the text in question. Vuillemot et al. [59] designed a system consisting of various visual interfaces for dynamic exploration of a single literary text: The Making of Americans by Gertrude Stein. Tag clouds and self-organizing graphs are used to explore character networks, to review vocabulary, and to filter by part of speech. Another technique applicable to digital humanities data is DocuBurst, a visualization that shows hierarchical summaries of a text in circular patterns [14]. A number of visual analytics approaches support the discovery of texts in a corpus that are related to a given problem statement. For example, Overview was designed to help investigative journalists find articles on specific subjects [10]. Texts are hierarchically clustered based on content similarity, and the system can be used for systematic search, summarization and annotation purposes, and for close reading of text documents. The visual analytics system presented by Heimerl et al. [25] uses interactively trained classifiers for document retrieval on large text collections. Koch et al. [42] designed a system with multiple views to browse a large collection of patents. A rather typical use case for the digital humanities is outlined by Correll et al. [16]. Given a large text collection, a text analysis environment helps to detect statistical patterns in the corpus interactively, and passages in texts can be displayed by relevance according to a user's preferences. VisArgue is a framework that provides visual analytics methods to support research on deliberative communication in political sciences by highlighting deliberative patterns in political discourses over time, speakers, and debates [18]. The text collection in our project consists of different versions of medieval French literary texts, for which we designed a visual analytics environment to generate and improve alignments of related verse lines using an interactive visual interface.

\subsection{Visualization of Text Editions}

Discovering and aligning related patterns among different text sources are typical tasks in digital humanities applications where visualizations aid analyzing such patterns. Some works focus on the detection and visual analysis of re-used text passages. Riehmann et al. [51] combined a stream graph and heat map representations to browse through plagiarized text passages in PhD theses. AbdulRahman et al. [3] use 2D pixelmaps to illustrate different text re-use structures in order to communicate perfect text re-use patterns, insertions, deletions as well as transpositions. Jänicke et al. [34] introduce a grid-based heat map to highlight texts that share systematic or repetitive patterns. Besides these rather generic forms of text re-use, many research questions in textual criticism focus on a comparative analysis of different versions of a text. Usually, two versions are placed side by side and related text passages are visually linked [27]; an example from journalism is the News Auditor [7]. Versioning Machine [1] and JuxtaCommons [62] are standard digital humanities tools for this task. Other approaches operate on more than two versions and provide several visual interfaces to analyze 
textual variation. ShakerVis offers a parallel coordinates view and a dot plot view to illustrate the similarity of sections in various German translations of Shakespeare's Othello [20]. Asokarajan et al. [6] use a pixel-based visualization to support the explorative analysis of variation in classical Latin texts. Jänicke et al. [33] present a distant reading approach to analyze dissimilarity among 24 English Bible translations. Based on the hierarchical structure of the Bible, heat maps show variations on book, chapter and verse level. In the latter case, an alignment on sentence or phrase level is given. Then, directed graphs - so called Variant Graphs — can be used to examine word-level differences. For example, the Word Tree aligns all sentences of a source text that share the same beginning in the form of a tree [61]. An extension of this idea is the WordGraph that visualizes the commonness of phrases containing one or more predefined terms [50]. TRAViz is a layout tailored to visualize characteristic features of Variant Graphs in order to support the analysis of orthographic and synonymous variations as well as word-level transpositions among multiple versions of a text [35]. A comparable approach is presented by Silvia et al. [56] who apply a storyline metaphor to Variant Graphs. The system presented in this paper provides three different simultaneous views on juxtaposed text versions, (1) a distant reading bar view showing global alignment structures, (2) a meso reading view showing local alignment structures among verse lines of multiple versions, and (3) a close reading pop-up view showing word-level alignments in the form of Variant Graphs. We discuss the importance of this intermediate form of meso reading in more detail in Section 3 below.

\subsection{Close Reading Visualizations}

The importance of close readings for digital humanities scholarship in order to build trust in explorative system environments as a form of verification of distant reading hypotheses is discussed in [32]. Usually, close readings preserve the structure and semantics of the text that can still be read on screen, and visualization is used to enhance the close reading experience with additional information. Goffin et al. [22] suggest the design and placement of so-called word-scale visualizations-small glyphs that impart additional information about fragments of the text in question. For example, small bar charts show the variants of observed words, or the background color of a word can be used to encode OCR certainty. AnnotateVis [12] instead mimics the traditional close reading process using computational means. The text is placed in the center and the screen margins to the left and to the right are used to position various types of annotations (notes, images, audios, videos, distant readings). Some close reading visualizations are designed to aid investigating certain features of texts, e.g., sound in poems. The tool Poemage [45] provides two close reading views for that purpose, (1) a poem view that shows possible phonetic features of words, and (2) a path view that sketches phonetic patterns occurring in the text. A comparable tool is the Poem Viewer [2], which uses visual attributes to encode phonetic units as well as phonetic and semantic relationships in a poem. Highlighting words in texts is a very basic form of visualizing annotations; guidelines for an appropriate implementation of this approach are given by Strobelt et al. [58]. For example, Serendip applies this method [5]. After topic modeling is performed on a large text collection, the words of a text in close reading mode are colored according to the determined topical relationships. Also, statistical information collected by crowdsourcing can be illustrated this way [60].

\section{Defining Meso Reading}

Our implemented visual analytics system can be situated within recent discussions in literary studies after the digital turn. The increase in computational approaches to analyzing text have sparked debates about the scale and precision with which the literary scholar operates. The term distant reading, has emerged within literary circles, probably in convenient contradistinction to more traditional, human-centered slow interpretation done on a limited number of texts known as close reading. In the context of visualization, the more abstract and data-oriented a visualization is the more it is said to be distant. Close reading visualizations provide micro views, enriching plain text with additional information yet keeping the text available for the human reader, whereas distant reading visualizations leverage text structure and semantics, providing macro views that emphasize statistical features of a text corpus, but usually eclipsing the text itself [32].

Literary criticism has come to recognize that close and distant approaches are combinable and perhaps exist more as a continuum than a binary, or more as complementary than an exclusive methodology $[38,53]$. Generally, this continuum exists in a number of visualization scenarios that implement the Visual InformationSeeking Mantra [54]: "overview first, zoom and filter, then detailson-demand." Overview stands for the macro view and details-ondemand for the micro view. Zoom and filter mechanisms span the in-between continuum providing manifold perpectives on the observed data for different zoom levels. By now, such a continuum for visualizing text does not exist-either a (single) text is readable or textual summary is visualized. In order to support the visual analysis of intertextual relationships, we assert that a third space of critic-centered text visualization is required for complex textual scenarios that we call meso reading. By meso reading we mean a visual strategy that ensures that distant patterns are discernable and passages of text are legible at the same time and within the same visual field for the human reader in order to assess the validity and importance of the pattern in context. In meso reading visualizations, the text and statistical features about the text occur together, providing the human reader with an interpretative frame. In our text visualization scenario, the meso reading view was required to assert the relative importance (or falsity) of computed alignments.

\section{Our Research on Textual Variance}

Our research is situated within a humanistic tradition of studying the transmission of texts via the multiple changes they undergo. Instead of restoring texts as nearly as possible to their original form [41], or even positing the existence of an Ur-text, we collect and compare as many of the versions of a specific work that are extant in the historical record. We do not know if these versions were copied verbatim from written texts, written down from memory or improvised based on some form of oral performance.

Comparing versions of a text The task of the textual critic is to observe all the changes in a text through its transmission. Text editors have favored very different theoretical approaches to "establishing the text," or what in common parlance was making a critical edition. Some have preferred the identification and comparison of different text versions aiming to reconstruct the archetype (the Lachmannian school) and others have chosen what was considered to be the best text, building their edition around one preferred manuscript (the Bédierist school). When a text was transmitted orally before being committed to writing, or even when it existed in writing, but authors continued to reinvent the work poetically, extant versions vary strongly. Our system allows the critic to explore different levels of text reuse and to hypothesize about the "movement" of texts.

Mouvance Swiss medievalist Paul Zumthor used the term mouvance to designate the high degree of instability in medieval text traditions, and to describe the mixture of textual, literary elements of written works with oral, performative ones resulting in highly variant readings and reworkings [63]. One famous example that exhibits a significant degree of textual and performative mouvance is the epic poem the Chanson de Roland, known to be transmitted in six major versions and three fragments, the first version dating from the early twelfth century. In order to discover related text fragments among these versions, the medievalist faces two major obstacles. 


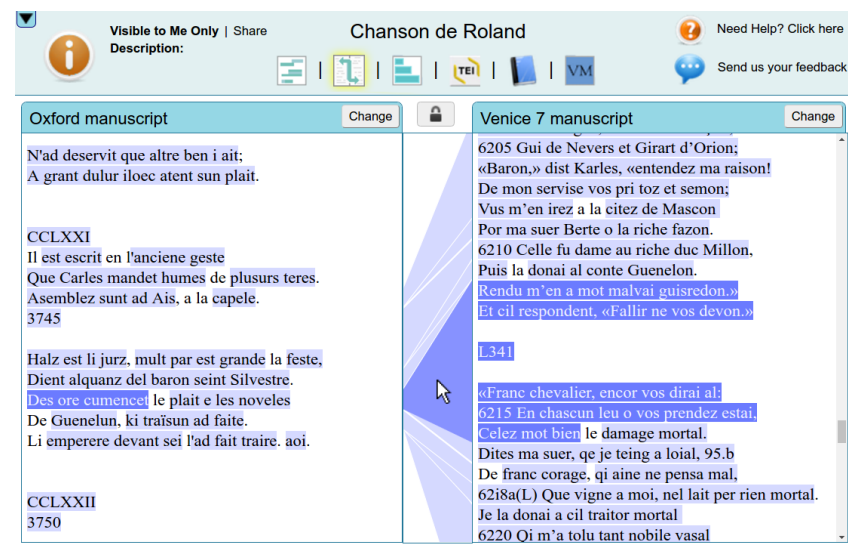

Figure 2: Juxta Commons alignment for two Chanson de Roland versions: numerous unrelated text fragments are aligned, an example is highlighted in dark blue.

First, the lengths of the versions vary greatly, ranging from 2,392 (Lyon manuscript) to 8,201 verse lines (Venice 7 manuscript). The oldest known version, the Oxford manuscript, has 4,002 verse lines. The second obstacle is the orthographic instability of the medieval French language. The same word can be spelled differently across the different manuscripts, but even within the same manuscript. Given these two major problems, a comparative close reading of different digitized versions with the aim of identifying related text fragments is a non-trivial task. There is so much variance that the patterns one ascertains "are too big to fit in a single reader's moving memory" [8]. Although this idea of a radical instability of texts named mouvance has been used to describe the situation of both pre-modern and digital textuality $[23,52]$, to our knowledge, it has never been used as a theoretical starting point for the comparative visualization of texts. Our visual analytics system supports the textual critic in the identification, as well as the analysis, of related text fragments for detecting and visualizing the mouvance of variant text traditions. It serves as a tool for hypothesis building about complex intertextual relationships.

Interdisciplinary Setting In order to ensure developing a beneficial, powerful tool that supports the given research task, we focused on a user-centered design approach $[4,26,46]$. Gibbs and Owens pointed out that this is especially important for interdisciplinary collaborations in digital humanities projects where visualization scholars "generally [neglect] the typical humanities user in their design" [21]. We took our own [28] and the experiences of other visualization researchers [32] who worked together with humanities scholars into account in order to avoid such pitfalls. What followed was an intense interdisciplinary exchange resulting in a number of prototypes that prepared the ground for the proposed visual analytics system.

First Prototypes The initial intention of the medievalist was to "leverage the conceptual progress of visualization in facilitating hybrid, on-screen, multi-scalar reading of computationally aligned text" in order to explore what it might tell him about orally-inflected poetry. Using digitized text versions, we wanted to compute alignments and to visualize the result in different fashions. At first, we considered using an out-of-the-box solution. We first turned to the TRACER ${ }^{1}$ tool designed for detecting re-used text fragments as it operates on arbitrary text corpora and it detects reuse at the level of a line. In order to resolve spelling variance, the TRACER reqires lemmatization lists, but appropriable solutions are not implemented. Some lemmatization lists for medieval French exist,

${ }^{1}$ TRACER tool: http://www.etrap.eu/research/tracer/ e.g., for the Chanson de Roland Oxford manuscript ${ }^{2}$ but their use is insufficient as they are based only on the oldest manuscript's orthographic conventions. As medieval French lemmatizers are not robust for all periods and dialects, our attempts at alignment were not successful. Our next attempt was trying standard digital humanities tools for our task, namely Versioning Machine [1] and Juxta Commons [62] - both yielding unsatisfactory results. The Juxta alignment visualization prototype is shown in Figure 2. Based on a diff component, the underlying algorithm inserts gaps so that the two versions' lengths are equal, and then defines areas of change disregarding larger amounts of inserted or deleted verse lines, ignoring transpositions altogether. The medievalist evaluated the results as nearly illegible, mentioning that the "central column does an insufficient job at expressing the complex instability of the medieval text tradition" [36]. No insight was gained into why certain pairs of lines had been aligned. Learning from the experience of these first attempts, we decided to closely interlock computational alignment and visualization in order to convey visually what had been achieved through the computational alignment algorithm. We then developed different prototype visualizations advancing our interdisciplinary thought process:

- Manual alignments: The medievalist prepared machinereadable, manual alignment tables for different source texts, for which the computer scientist developed a visual representation [37]. This was an important initial step for the project. On the one hand, the medievalist's choices exemplified the human thought process that leads to alignment, and that could basically be translated into parameters to be defined for the computational alignment approach. The medievalist was left wondering on what basis an ideal alignment might be made: on repeated words, half lines, full lines or even multiple line chunks. We realized that it ideally would capture all of the above. The computer scientist, keeping the user's concerns in mind, worked to transmit the potential of visualization to reflect the "complex instability" of medieval texts.

- Duggan's formulas: In 1969, a leading scholar of the Chanson de Roland Duggan published a concordance of the Oxford manuscript [17]. The concordance allowed him to study the phenomenon of repetition, albeit based on a single manuscript, and using it he published a monograph-length work on formulaic speech in the Chanson de Roland (battles, persons, epithets, acts of speech, etc.). His work was foundational, as he meticulously analyzed repetition in the medieval French text. Not only did he never "see" the resultant formulas visualized. The medievalist sought to create a visual representation of Duggan's taxonomy of repetition, as a way of deciding if two verse lines containing the same Duggan formula should be aligned or not. Although we permitted a slight edit distance for the discovery of the formulas in the Venice 7 manuscript, the shorter Oxford manuscript has double the number of exact matches. As this distorts the bigger picture, we decided to use Duggan's formulas only as a category for alignments to be stored, not as an indicator. What Duggan achieved with the Oxford manuscript alignment would not help us across the entire textual tradition.

- Computational alignments: In order to set up a list of parameters to be considered when computationally determining variant verse lines between two text versions, we developed a series of prototypes using different parameter settings, e.g., exact vs. fuzzy match, or the exclusion of a custom stopword list from the computation process; the resultant parameter list is discussed in the following section. Meso reading visualizations were designed to analyze variance at the level of the 
line, but when exploring results using the prototypes, the medievalist expressed an interest to analyze alignment patterns on different text hierarchy levels.

List of Requirements Discussing the various prototype implementations were of mutual benefit to both scholars' thought processes. On the one hand, the computer scientist was able to seize the properties of variance that yield relevant alignment results, and to design and evaluate visual metaphors for reflecting textual similarity. On the other hand, the medievalist came to appreciate the potential of computational alignment and visualization, and both their benefits and limitations. With these insights in mind, we derived a list of major system requirements to meet the needs of the medievalist analyzing variance among medieval French text versions:

i. Dynamic parameter setting: The medievalist mentioned the need for "multiple visuals" both to grasp how the alignment changes with different parameters, and to single out their individual influence on the result. The final list of parameters to be adjusted for the computational alignment are explained in detail in Section 5.3.

ii. Multi-level visualization of textual variance: In order to meet the medievalist's desire to analyze "both stability and variance among the text versions" on different text hierarchy levels, we provide a distant reading view to explore the entirety of computationally gained alignments on the document level, a meso reading view to inspect patterns on line level, and a close reading view for word level alignment. The views and their linkage are discussed in Section 5.4.

iii. Visual analytics approach: In order to realize to "visually solidify mouvance," we implemented a visual analytics system that allows dynamic manipulation of parameters, updates the visual representation of the alignment according to these changes, and provides a mechanism to make individual alignments persistent - thereby recording the properties of true positives, but also considering alignments rated as false positives. Detailed information about the presented visual analytics system can be found in Section 5.5.

\section{Interactive Visual Text Alignment}

According to the taxonomy of text analysis tasks provided by the Visual Text Analysis Process typical for digital humanities projects [32] our work falls into the subcategory text version comparison of the main category similar patterns. Our main goal was to design a system that provides a novel workflow to align highly variant medieval text versions.

\subsection{Text Sources}

Our usage scenarios (see Section 6) narrate visual text alignment procedures for medieval French text traditions. All texts-attached with a dating important for medievalists to hypothesize possible lines of transmission-were given as plain text files in a line-separated format. In a preprocessing step, special characters are removed. In addition, we removed diacritics since they are not original to medieval writing, but rather editorial additions. As editorial conventions in assigning such diacritics fluctuate, considering diacritics complicates the discovery of variant spellings necessary for the alignment algorithm.

The goal of our system is supporting the comparative analysis of text versions based on the similarity of verse lines. Therefore, as illustrated in Figure 3, we first regard each text edition as a set of sequentially ordered lines. As the alignment algorithm proposed in Section 5.3 uses a Variant Graph structure, each line $X$ is considered as a sequential order of words $X=x_{1}, \ldots, x_{|X|}$ in the form of a directed path using words as vertices. An individual alignment is a
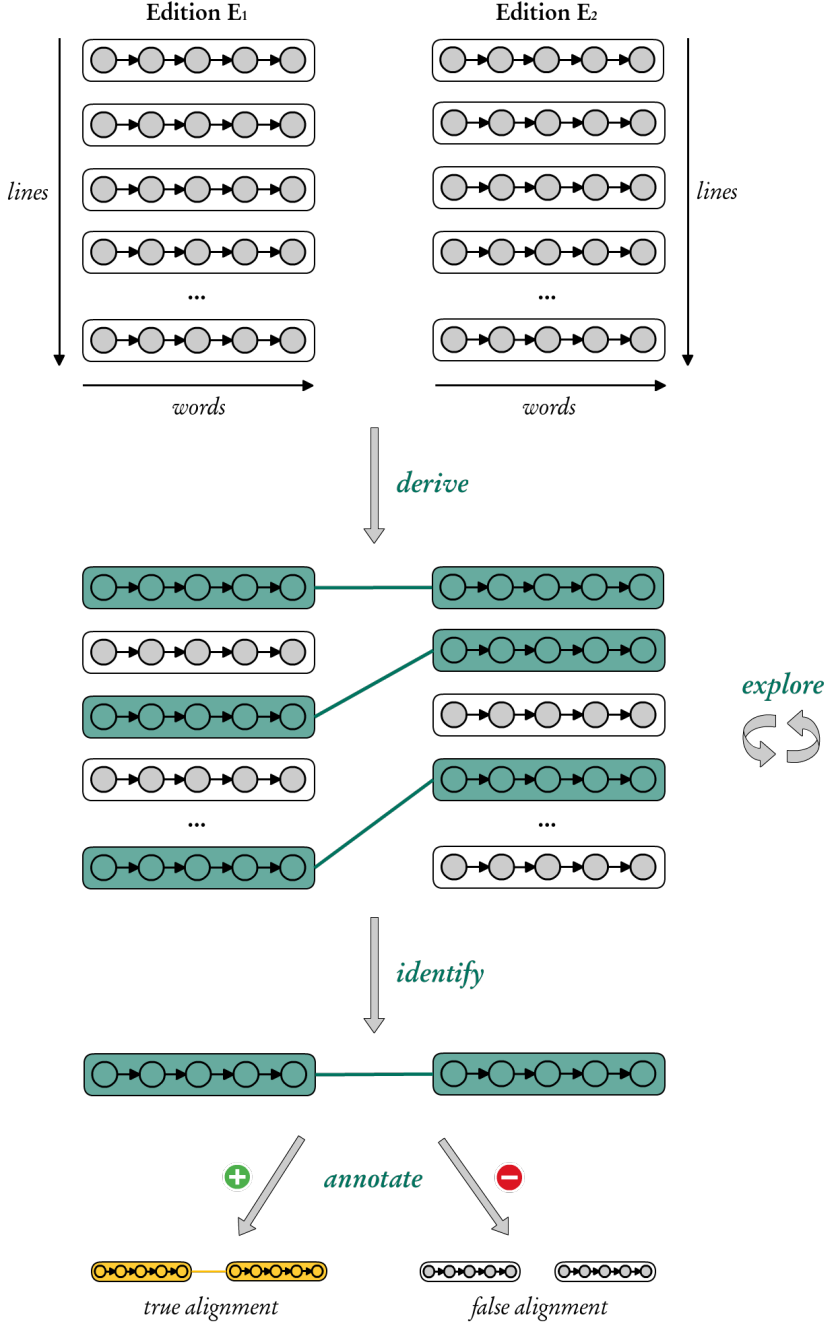

Figure 3: Data \& task abstraction.

relation between two verse lines $X$ and $Y$ belonging to different text versions.

\subsection{Text Analysis Task}

The domain-specific text analysis task is the discovery of both instability and repetition in poetic language, more precisely, among highly variant, orally-inflected medieval texts. The medievalist required a system (1) to analyze how similar the given text versions are, (2) to discover verses that are shared among the versions, those ones that were added and those ones that were left out, and (3) to assemble a interactive alignment for two versions under investigation.

Our system is designed to support these tasks by a semi-automated process. Following Munzner's guidelines for task abstraction [47], the domain-specific tasks can be abstracted as illustrated in Figure 3. First, for two juxtaposed text versions the visual analytics system automatically derives relations among lines that are categorized as similar according to the parameter-driven alignment algorithm outlined in Section 5.3. Then, the result is explored in order to detect patterns and discover specifics. In this process, individual relations need to be identified, and to achieve the targeted "perfect alignment" they are annotated as true or false alignments. 


\subsection{Data Transformation}

The purpose of the data transformation step is computing an alignment for two given versions $E_{1}$ and $E_{2}$ that delivers relations $\left\{X, Y \mid X \in E_{1} \wedge Y \in E_{2}\right\}$ between significantly similar verse lines $X$ and $Y$. We define $|\{X, Y\}|$ as the length of an alignment, which is the total of found word matches. Example alignments of medieval French verse line variants are given in Figures $4 \mathrm{a}$ and $4 \mathrm{c}$.

Alignment While various algorithms are applicable to align $X$ and $Y$, e.g. sequence alignment methods from bioinformatics [48, 57], we apply a text re-use alignment algorithm [29] that serves as the basis for a variant graph visualization and achieves good results [35]. As a brute force approach, this method yields an alignment sequence with the maximum possible number of in-order matches. For the example in Figure 4a, the text re-use alignment algorithm computes the green colored matches $\left\{x_{1}, y_{1}\right\},\left\{x_{2}, y_{2}\right\}$, $\left\{x_{3}, y_{3}\right\},\left\{x_{5}, y_{6}\right\}$ and $\left\{x_{6}, y_{7}\right\}$. As variant graph structures are directed and acyclic, the algorithm neglects transpositions. In order to improve the n-gram and coverage scores (see configuration below) for $X$ and $Y$, we perform a post-processing step that looks for matches among the unaligned words, which yields the additional pairs $\left\{x_{4}, y_{8}\right\},\left\{x_{7}, y_{4}\right\}$ and $\left\{x_{8}, y_{5}\right\}$ in the above example. We do not consider stopwords in this step since the medievalist faced numerous false positive alignments, which distorts the visual representation and interpretation of mouvance, an example is shown in Figure 4b.

Configuration An important requirement for development was designing a system that communicates all aspects of data transformation, so that the medievalist "is able to generate trustworthy hypotheses" [32] about mouvance when exploring the results visually. The medievalist did not want to work with a black-box [21], and when developing prototypes during the first project period he asked for various representations reflecting different configurations for the alignment computation in order to be able to assess the effect of individual parameters on the visualization of mouvance. In other words, he wanted to steer the computational alignment by redefining parameters on demand in order to update the visual representation aiming to generate knowledge - this demanded capability is well defined as the Visual Analytics Process by Keim et al. [40]. Therefore, we provide four interdependent parameters to be interactively modified by the user:

- Edit Distance: Because of the highly inflected and orthographically unstable language at hand, variant spellings needed to be taken into account during the alignment procedure. Two words $x[1 \ldots|x|]$ and $y[1 \ldots|y|]$ are considered as spelling variants if

$$
x[1] \equiv y[1] \quad \text { and } \quad \phi \leq 1-\frac{\operatorname{lev}(x[2 \ldots|x|], y[2 \ldots|y|])}{\max (|x|,|y|)} .
$$

The first condition to be fulfilled is the equality of the first letters of $x$ and $y$. For the remaining parts of $x$ and $y$, the string similarity (normalized edit distance) [13] must not be smaller than a user-configurable threshold $\phi(0.5 \leq \phi \leq 1)$; we chose $\phi=0.5$ as least necessary similarity as the overall number of alignments was too high for lower values due to numerous false positive spelling matches. Only considering the latter condition was insufficient as also rhyme patterns-irrelevant for the given text analysis task-were considered as spelling variants. In Figure 4a, the similarity for bras and braz is 0.67 , the similarities in Figure $4 \mathrm{c}$ are 0.75 for clost and clos and 0.89 for avenanment and avenaument.

- N-grams: The user can configure the minimum required ngram size $n(2 \leq n \leq \omega)$ that is the largest number of subsequent word matches in $X$ and $Y$. Whereas at least bi-grams in $X$ and $Y$ are necessary to consider $\{X, Y\}$ as an alignment, the maximum possible value $\omega$ for $n$ depends on the longest

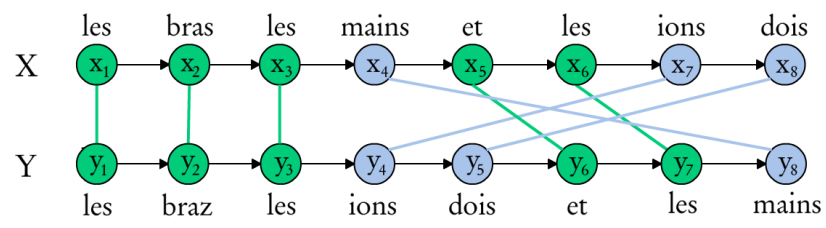

(a) Perfect alignment of two variants; the applied text re-use alignment algorithm delivers the "green sequence," then, the transposed blueish matches are added.

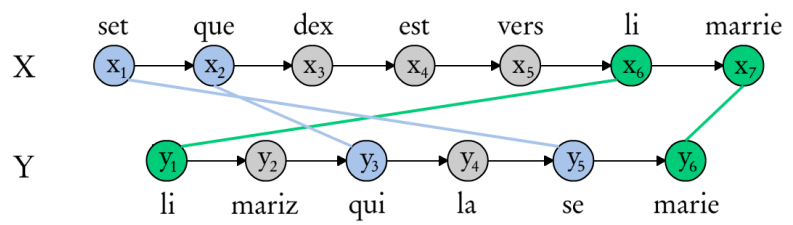

(b) False positive alignment if transposed stopwords would be considered.

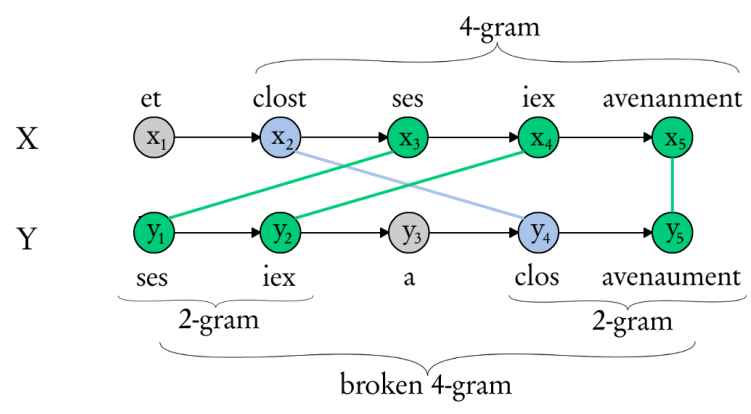

(c) N-grams and broken n-grams.

Figure 4: Example alignments of medieval French verse line variants.

word sequence of a verse line in the currently juxtaposed text versions $E_{1}$ and $E_{2}$. In the example in Figure $4 \mathrm{c}, X$ contains a 4-gram and $Y$ a 2-gram, so that $\{X, Y\}$ is only aligned if $n=2$.

- Broken n-grams: Quite often, the only difference of an alignment candidate $\{X, Y\}$ is a single word in the middle of $X$ and/or $Y$ that is either inserted, synonymous, or a transposed stopword. Then, large n-grams cannot be achieved. In order to not turn those alignments into false negatives, the user can allow for broken n-grams, which is the total number $|\{X, Y\}|$ of word matches in $X$ and $Y$ each. Considering broken n-grams in the example in Figure 4c, $Y$ contains a broken 4-gram, so that $\{X, Y\}$ is aligned if $n \leq 4$.

- Coverage: In order to ensure that a specific proportion of words of $X$ and $Y$ are aligned, the user can configure a minimum coverage value $\psi(0.2 \leq \psi \leq 1)$, so that the following requirements must be fulfilled:

$$
\psi \leq \frac{|\{X, Y\}|}{|X|} \quad \text { and } \quad \psi \leq \frac{|\{X, Y\}|}{|Y|} .
$$

$\mathrm{N}$-gram and coverage settings are logically linked. If the chosen n-gram is too large, short verse lines may not be considered as alignments, especially if stopwords are excluded. To overcome this problem, we link $n$ and $\psi$ with a logic OR if $n \geq|X|$, respectively $n \geq|Y|$. On the other hand, for a small n-gram value the probability that unrelated long verse lines are considered is high, especially when stopwords are not excluded. To handle such cases, we link $n$ and $\psi$ with a logic AND if $n<|X|$, respectively $n<|Y|$. 
Complexity The computational effort of the alignment algorithm can be assessed by $\Theta\left(\left|E_{1}\right| \cdot\left|E_{2}\right| \cdot k\right)$, with $\left|E_{1}\right|$ verse lines in $E_{1}$, and $\left|E_{2}\right|$ verse lines in $E_{2}$. For example, the juxtaposition of the Oxford manuscript (4,002 verse lines) with the Venice 7 manuscript (8,201 verse lines) of the Chanson de Roland requires 32,820,402 alignment computations. The value of $k$, dependent on $|X|$ and $|Y|$, stands for the average effort undertaken for the alignment of the two verse lines $X$ and $Y$. Re-configuring the string similarity threshold $\phi$ requires re-computing the entire alignment as it may significantly change. In order to facilitate an efficient work with a responsive system, alignment sets are pre-computed for all possible $\phi$-values. Such an alignment set contains all aligned verse line tuples sharing at least a 2-gram and having a minimum coverage of $\psi=0.2$. In the above mentioned Chanson de Roland scenario, we receive 226,129 alignment tuples for the minimum string similarity $\phi=0.5$, and the remaining effort is narrowed down to filtering out those tuples that do not reach the necessary minima for $n$ and/or $\psi$.

\subsection{Visual Text Analysis}

While two versions are typically compared in a juxtaposing view [1, $62]$, we extend the dimensionality to an arbitrary number of versions limited first and foremost by the screen's width. To support a multi-level visual analysis of the gained computational alignment(s), we provide views for three different texts hierarchy levels: whole text versions, verse lines and words. On all levels, our design facilitates discovering transposed text fragments [32] integral to a textual scholar's work. Examples for all views can be found in Figure 6.

Distant Reading To produce a rough overview of alignment patterns throughout the observed text versions, we draw a miniature representation for each version in the form of a vertical bar reflecting its number of verse lines in contrast to the other shown versions. Computed alignments are drawn as polylines along the vertical bars so that alignment patterns are made visible. For example, multiple subsequent parallel polylines indicate aligned paragraphs whereas transposed text fragments lead to polyline crossings. Via mouse click on a vertical bar, the user jumps to a desired section of the corresponding version, which also updates the meso reading view.

Meso Reading According to the Visual Text Analysis Process [32], applied visualization techniques are grouped into close and distant readings. As we have explained above, there is a "reading level" between these two scales of visualization that we call meso reading. Since multiple texts are displayed, in what textual scholars call synoptic views, the visualization is able to convey more complex patterns of textual relationship across versions that are impossible to be visualized in a single text view. A meso reading might be said to connect multiple close readings all the while transmitting information that lies beyond the scope of a close reading. It might also be said that meso reading serves as a zoomed version of the distant reading visualization. Here, we use the intuitivity of stream graphs [11] to connect aligned verse lines among different versions. Seen as the most important component of our system to visualize mouvance, the meso reading needs to be legibile, comprehensible and interactive. Semi-transparent streams ensure that crossing streams (transposed verse lines) visually separate from each other. Furthermore, repetitive aligned verse lines (e.g., those containing a Duggan formula) stick out due to more saturated background colors. When two versions are juxtaposed, tiny previews of the word level alignments are shown next to each line that is part of at least one alignment tuple in the form of heat maps reserving a small rectangle for each word of the line. Stopwords are indicated with an "S," and the words that contribute to the alignment are colored (again, saturation highlights the frequency of reused words). For a more detailed inspection of an individual alignment, clicking on a stream opens a popup window for close reading at the level of a particular line.
Close Reading Next to plain text, the close reading view provides word level alignments for the corresponding verse lines in the form of two Variant Graph visualizations [35]. The first graph is drawn without applying an edit distance measure, the second graph is built according to the current string similarity configuration. The medievalist wanted both graphs to be juxtaposed in order to get an immediate justification for the computed alignment of variant spellings. The second graph is interactive, so that the alignment structure can be modified, e.g., variants of a word that were not aligned by the computational process can be manually aligned. Within the close reading view, individual alignments can be confirmed-optionally, an alignment category can be set, e.g., Duggan formula, perfect match, frontal hemistich)—, so that it gets persistently stored in the backend. Furthermore, an equivalency table for spelling variants is generated in the background according to the merged nodes (identified spelling variants) of the second variant graph. On the one hand, we use the equivalency table for future alignment computations as it supersedes the necessity of a lemmatization list. On the other hand, this leads to a sustainable benefit for medievalists requiring such a dictionary.

Multi-version View When computational alignments are stored, usually, the medievalist focuses on only two text versions which are clearly presented in a juxtaposed view. But, observing and confirming more than two versions at once (cf. $[15,30])$ quickens the comparison task. Initially, we order the text versions so that the number of streams crossing unrelated texts in the meso reading view is minimal. When such crossings yet occur, we render them with a high transparency. Furthermore, versions can be ordered according to the given datings, so that mouvance can be visualized as a function of time, and possible lines of influence can be hypothesized. Figure 1 shows the alignment of three juxtaposed versions of La vie de sainte Marie l'Egyptienne.

\subsection{Visual Text Alignment Workflow}

Though the Visual Text Analysis Process [32] does not explicitly discuss the necessity of iterative interaction with the data, their transformation and visual representations in order to gain insightdue to the fact that complex systems are often seen as "black boxes" by humanities scholars impeding their adoption [21], the value of visualization is often only the capacity to serve as evidence in digital humanities inquiry-, the Visual Text Analysis Process model still admits the implementation of a visual analytics feedback loop [40].

Alignment Workflow After selecting two text versions to be juxtaposed, a typical workflow of the medievalist starts with an initially suggested parameter setting derived from the features of the already stored alignments $S$ for the juxtaposed versions. For example, we define the initial coverage value $\psi_{s}$ as

$$
\psi_{S}=\min _{\{X, Y\} \in S}\left(\min \left(\frac{|\{X, Y\}|}{|X|}, \frac{|\{X, Y\}|}{|Y|}\right)\right)
$$

to prevent newly computed alignments from having have a lower coverage than the confirmed ones. The other parameters are initialized similarly. After computing the alignment according to the said configuration, the results are visualized in the form of lines in the distant reading view, and in the form of streams in the meso reading view. Then, typically an alignment session starts where the medievalist focuses first on the meso reading view to search for alignment tuples to store. The scholar can also create an alignment tuple that have not been discovered by the alignment algorithm. With each stored tuple, the user is provided with suggestions for each parameter setting if it should be increased or decreased, dependent on all stored alignments. After parameter changes and re-computing the alignment, streams are updated according to the color schema shown in Figure 5. As such, the medievalist is able to understand 
alignment detected in current iteration

alignment of the last iteration confirmed by current iteration

alignment of the last iteration not confirmed by current iteration

stored alignment not confirmed by current iteration

stored alignment confirmed by current iteration

Figure 5: Stream coloring according to the current alignment iteration.

how parameter changes affect the resultant alignment traceable in the meso reading view.

\section{Usage Scenarios}

The three text traditions employed here include Chrétien de Troyes' Perceval: le conte du Graal, the Chanson de Roland as well as a saint's life, La vie de sainte Marie l'Egyptienne. Each usage case represents a very different textual record, by which we mean that multiple copies of the text written down in manuscript at very different moments of time and in different places. Each usage case also provides different examples of repetition across text traditions, what in literary studies can be called the problem of intertextuality, that is, how one text is interwoven with text, or simply language, from somewhere else. The three examples below illustrate how the resultant visualizations helped the medievalist think about the the problem of variance across genres.

Perceval Perceval is the fifth work of the twelfth-century romancer Chrétien de Troyes, composed between 1182 and 1190. It is a verse narrative written in octosyllabic verse and it is extant in some twenty different manuscripts. The genre attributed to this text is romance. Romance is a more or less stable form, by which we mean that, across versions, while there may be slight interpolations of passages, variant words and expressions, the different versions can be humanly collated with relative ease. This means that computational collation is easy as well, although the initial configuration considering alignment tuples sharing broken 3-grams delivered a sparse alignment. By decreasing the minimum string similarity to $\phi=0.6$ for correction of spelling variance, the medievalist received a very dense alignment with numerous repetitions. Both disallowing broken 3 -grams and changing the minumum coverage value $\psi$ from 0.4 to 0.6 lead to a sufficient alignment (see Figure 6a). Looking at the result, one gets a flavor of just how close the texts are. The versions are perhaps so well aligned though that we tend to think of them as two near equivalent versions. But this is to miss the reuse of repetitive formulaic speech in romance. As the crossing streams attest, there is also a considerable amount of computationally detected repetition, not between different versions but within the same poem that needs to be analyzed further. During the session, false alignments were also discovered. For instance, "Qui me dist que li ange sont" and "qui me dist que meisons estoit" are both examples of descriptive indirect speech, an important phenomenon in the texture of romance. The two lines do not have the same meaning at all. Thus, this alignment tuple is not aligned in the collation session. The meso reading view also shows numerous correctly aligned full-line matches. Although this is not what we set out to do with the example of Perceval, our system identified a previously unnoticed stratum of full-line repetitive discursive language that is part of the warp and weft of romance. This is a fascinating discovery and a reminder to the literary historian of the existence of this kind of language patterning in romance.

La Chanson de Roland When compared with the various copies of the Perceval romance, the Chanson de Roland - epic poetry in medieval French - could not have a more distinct composition style across its versions. In order to get the alignment shown in
Figure $6 \mathrm{~b}$, the medievalist decreased the required string similarity to $\phi=0.5$. The criss-cross of repetitive formulaic language we saw in romance is present, but here it is not embedded in a basically aligned narrative structure like in the case of romance. Focusing the meso reading visualization, the medievalist discovered a very different kind of alignment. First of all, the rectangular heat maps illustrate many more partial line repetitions, confirming what generations of scholars have suspected about the medieval French epic, but have not had the means to visualize and prove fully. Furthermore, one of the important features of medieval French epic is the distribution of those fragments bidirectionally in the poem as the continually crossing lines in the distant reading and streams in the meso reading views illustrate. Figure $6 \mathrm{~b}$ shows examples of partial-line formulaic fragments with very different frequencies, e.g., Duggan's battle formulas "si vait ferir" and "el cors li met." Unlike in the case of romance, epic texts do not necessarily align semantically. Medieval French epic is structured using a deep stratum of oral language that needs to be studied in greater depth.

La vie de saint Marie l'Egyptienne The third example is that of a saint's life, a kind of story that was retold in very different ways across a tradition. Saints' lives are typically made up of a set of basic narrative segments, like building blocks that are put together sequentially by others but with different inflection. Different poetic versions of the legend are of divergent length and style. One particular example illustrates the capacity of our system to chart the way that a legend is rewritten over time. Figure $6 \mathrm{c}$ illustrates the version written by Rutebeuf in the thirteenth century aligned with the version inserted into the longer satirical work known as the anonymous Renart le Contrefait of the first half of the fourteenth century. The comparison is not a trivial one, since the two authors both employ a polemical tone and draw upon satirical anti-clerical themes. As is obvious from the distant reading view, the later Renart le Contrefait is a significantly shorter text than Rutebeuf's version of the legend. With the default setting, numerous unrelated lines were connected. Requesting 4-grams instead of 3-grams filtered out many false positives and revealed a special version of intertextuality based on sequential narrative borrowing and rewriting - the Renart le Contrefait version is actually a condensed version of segments of the Rutebeuf legend with full-line or near full-line borrowings from the latter.

Summary The tapestry of repeated segments across the different related versions in various genres (romance, epic and hagiography) display distinct patterns. Put another way, mouvance cannot be quantified and visualized in the same way across all textual scenarios. It is not a theory of textual instability that can be strictly formalized, but rather a text-specific phenomenon that can be modeled iteratively in our system. The visual analytics system allows parameter-driven exploration and correction of the computational alignment, but also as in the three cases mentioned above, it provides very promising avenues for future literary research concerning the comparison of related texts. Since the borrowing in the last example is so flagrant and based on full-line repetition, the idea of user correction is less useful than in the previous examples, but having built the system upon the assumptions of the most complex patterned texts has proven to be a valuable exercise. It allows for the most complex textual systems to be scored. Simpler alignment systems such as Versioning Machine and Juxta Commons cannot account for such complexity and multifaceted alignment.

\section{InTERdisciplinary Design Process}

The proposed visual alignment system was designed to support the medievalist in analyzing and aligning highly variant text traditions, an onerous task without computational means. With the experiences and insights gained when preparing prototypes, we iteratively developed the system proposed in this paper. Likewise, the medievalist 


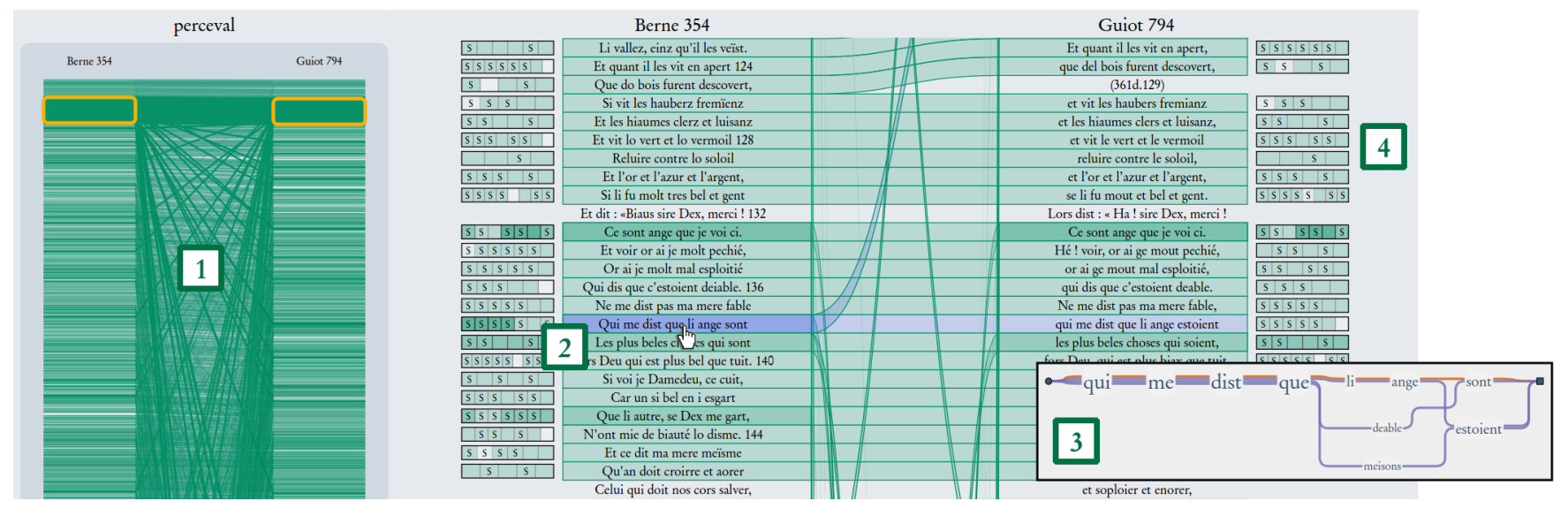

(a) Juxtaposed Perceval versions: (1) distant reading shows a high similarity in the form of parallel lines and a few repetitions as diagonal lines, (2) meso reading of the repetitive verse line "Qui me dist que li ange sont", (3) close reading reveals false positive alignment, (4) numerous full-line matches.

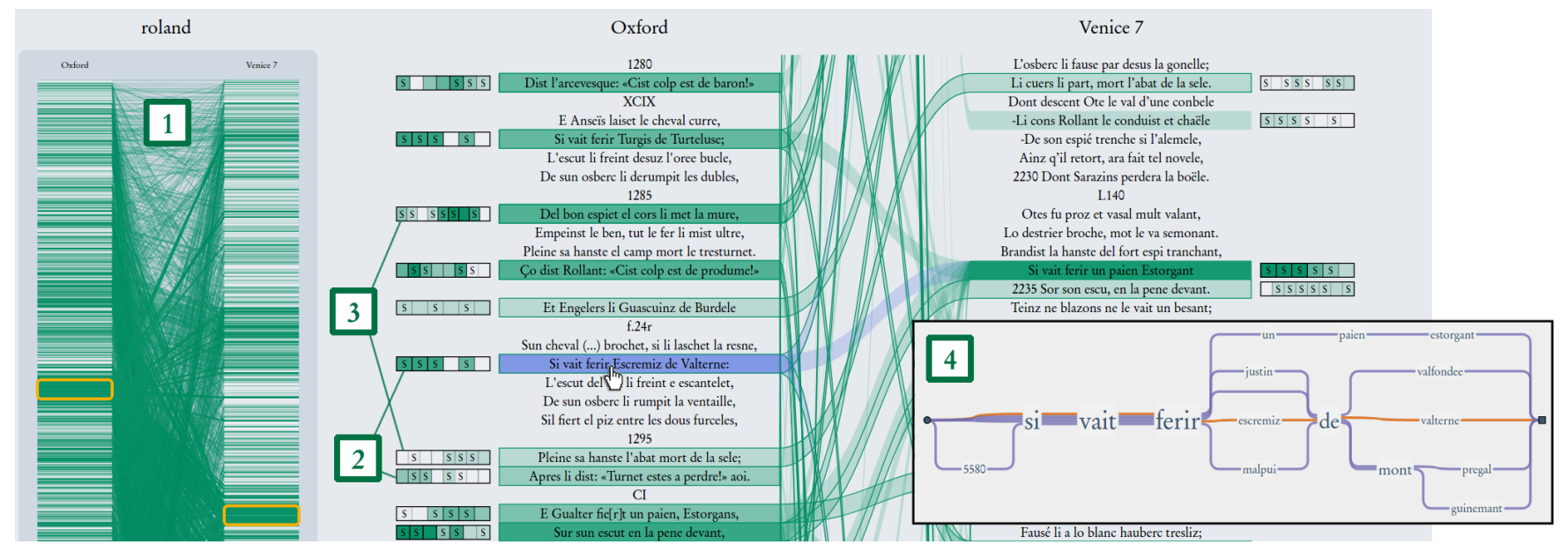

(b) Juxtaposed Chanson de Roland versions: (1) few parallel and numerous diagonal lines in the distant reading illustrate the instability of versions, (2) examples of frontal half-line matches, (3) examples of posterior half-line matches, (4) close reading of verse lines containing the Duggan battle formula "si vait ferir".
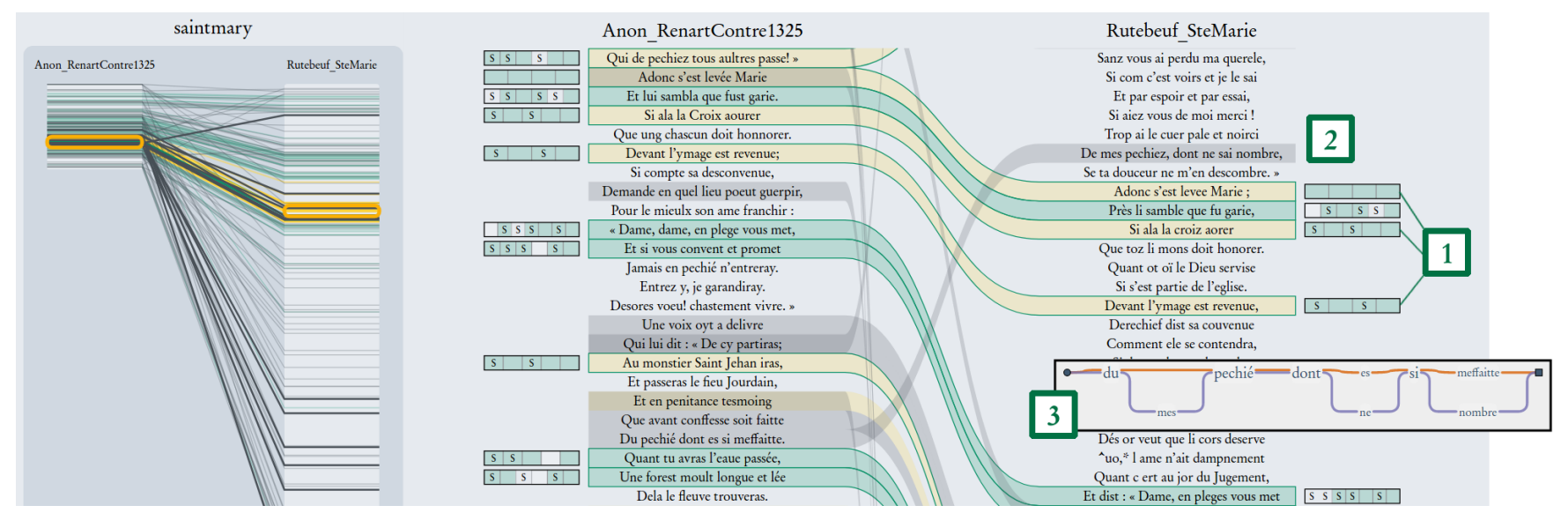

(c) Juxtaposed La vie de saint Marie l'Egyptienne versions: (1) full-line matches annotated as true alignments, (2) requesting 4-grams removes false positive alignment, (3) close reading of unrelated verse lines.

Figure 6: Usage scenarios of our visual analytics system. 
iteratively evaluated current milestones, so that we could consider his feedback for future developments.

Alignment Algorithm Implementation The medievalist began working with the Perceval example where it was easy for him to visually verify precision and recall since the Perceval versions display the typical properties of orthographical instability, but with very similar structures and lengths. At first, this gradually improved the proposed alignment algorithm. Initially, we provided two alignment strategies; an ordered solution without considering transposed words, and a MinHash technique ignoring the order of word matches. The medievalist evaluated and compared the results of both alignment algorithms using the meso reading view to get an overview and the close reading view to inspect individual alignments. $\mathrm{He}$ inferred that the ordered solution had a high precision and a low recall, the MinHash method the contrary. After a joint analysis of the reasons we combined both strategies in order to gain results that meet scholarly expectations as both precision and recall increased. When introducing edit distance into our alignment procedure, we prepared lists of word tuples that matched for different string similarity thresholds, so that the medievalist could get an idea how this measure works. He remarked that the precision when aligning the Perceval versions was low as often rhyming words with strongly varying word beginnings - irrelevant for the collation task-were taken as variant writings. We first applied different string similarity thresholds for word beginnings and endings leading to a lower recall. Finally, when taking first letter equality as mandatory he assessed the results as satifactory. For the implementation of broken n-grams, we initially suggested to allow for a flexible definition of the degree of brokenness. In order to keep the parameter spectrum humanly processible, the medievalist advocated a simple boolean solution. We furthermore provided the parameter option to exclude stopwords from the alignment computation in order to get more precise results. But the observed user behavior showed that stopwords were always taken into account as excluding them usually delivered few results, so that we removed this parameter from the configuration interface.

Visualization Design The medievalist's feedback on the proposed multi-level alignment visualization was especially valuable to spot weaknesses of current prototypes. This included both aspects of visual representation and interaction design. First, this led to the inclusion of the close reading view as the medievalist desired to analyze individual computationally gained alignments on word level. He further asked for providing two Variant Graph versions in order to better understand how the alignment algorithm operates. Also, iteratively designing the meso reading view was of particular importance. Working with the first meso reading prototype, the medievalist struggled with the interpretation of the overall set of computed alignments as different types of line reuse occur, e.g., partial line reuse (frontal or posterior hemistich), ${ }^{3}$ complete matches, or broken n-grams. In order to more quickly perceive the type of line reuse, we designed word-level alignment previews, which turned out to be a key feature for the medievalist when using the system as neither printed versions nor out-of-the-box solutions do account for this level of textual concordance. Another important design aspect was implementing the color coding of streams. After providing means to configure the alignment computation, the medievalist desired to see how parameter changes effect the result. In collaboration, we selected colors from qualitative color maps [24] that suitably reflect the type of alignment.

The above outlined cases narrate the importance of involving the user in all development stages in order to implement design changes based on the received feedback aiming to optimize the value of the system. The medievalist assesses the results gained by the visual analytics approach to computationally align medieval French text versions as "surprisingly good," impossible to replicate manually.

\footnotetext{
${ }^{3} \mathrm{~A}$ hemistich is a four or five syllable half-line.
}

This underpins the benefit of our method for textual scholarship. But, due to the nature of the input data, methodological aspects of the text-critical process and the parameter-driven system design there are some limitations to consider.

Limitations Due to orthographical and synonymous variances, a single configuration that both maximizes precision and recall does not exist. The results for the Perceval versions illustrate, however, the efficiency of our approach to approximate a "perfect alignment." Even though the system is designed to expedite comparison for textual critics, retrieved alignment tuples still have to be examined individually. According to Bradley et al. [9], this fact situates our system within the concept of "slow analytics," a process where information visualization methods can support sense making in literary studies by means of repetitive decision making. Finally, the system was designed together with only one medievalist although other medievalists have experimented with it. Even though our approach reflects the fruitful collaboration between literature and computer science, it remains to be seen how literary history will receive our system and its expansion of Zumthor's concept of mouvance beyond a purely theoretical notion to an empirically parameter-driven and visualizable textual phenomenon.

\section{Conclusion}

Scholars have suggested that oral-like language is integral to the process of poetic creation for medieval texts as we have seen in the case studies above. This oral inflection causes not only different kinds of variance, e.g., orthographical and synonymous variance, transposition of words and verse lines, inserted paragraphs or reorganized rhyming structures, but patterns of variance are also not uniform across a text. As a consequence, the discovery of related text passages is a very confusing task for the textual critic to manage. Now, with the help of our visual analytics alignment approach, the alignment algorithm is capable of handling the mentioned heterogenous forms of variance, and the visualization assembles them empirically in a panoptic view.

The outlined visual analytics method was developed in close collaboration of a computer scientist and a medieval literary historian over an extended period following a user-centered approach. In the end, the medievalist finds a valuable instrument that yields "an alignment not to be understood as a final product, but rather a process, for understanding variant text traditions, supporting the generation of new hypotheses about textual behavior." He argues that the results gained with our approach could even replace the archetype concept for the investigated text genre-a hypothesis yet to be discussed by medieval scholars. On the other hand, the computer scientist was able to establish a computational alignment algorithm for highly instable text variants coupled with multi-level visualizations in a novel visual analytics environment that allows for an iterative improvement of alignment results, and in addition, for the gradually improvement of comparison of different text versions. In addition to typical close and distant reading views, we introduce an intermediate meso reading view that juxtaposes different text versions the way that the texts can still be close read, but it furthermore visualizes interdependencies of verse lines across manuscript versions. Although we designed the system for medieval French texts, our approach is adaptable to other languages or textual scenarios having the same heterogenous forms of variance. 


\section{REFERENCES}

[1] Versioning Machine, 2017. http://v-machine.org/ (Retrieved 2017-02-07).

[2] A. Abdul-Rahman, J. Lein, K. Coles, E. Maguire, M. Meyer, M. Wynne, C. R. Johnson, A. Trefethen, and M. Chen. Rule-based Visual Mappings-with a Case Study on Poetry Visualization. In Computer Graphics Forum, volume 32, pages 381-390. Wiley Online Library, 2013.

[3] A. Abdul-Rahman, G. Roe, M. Olsen, C. Gladstone, R. Whaling, N. Cronk, R. Morrissey, and M. Chen. Constructive Visual Analytics for Text Similarity Detection. Computer Graphics Forum, 2016.

[4] C. Abras, D. Maloney-Krichmar, and J. Preece. User-centered Design. Bainbridge, W. Encyclopedia of Human-Computer Interaction Thousand Oaks: Sage Publications, 37(4):445-456, 2004.

[5] E. Alexander, J. Kohlmann, R. Valenza, M. Witmore, and M. Gleicher. Serendip: Topic Model-Driven Visual Exploration of Text Corpora. In Visual Analytics Science and Technology (VAST), 2014 IEEE Conference on, pages 173-182, Oct 2014.

[6] B. Asokarajan, R. Etemadpour, J. Abbas, S. Huskey, and C. Weaver Visualization of Latin Textual Variants using a Pixel-Based Text Analysis Tool. In N. Andrienko and M. Sedlmair, editors, EuroVis Workshop on Visual Analytics (EuroVA). The Eurographics Association, 2016.

[7] M. Behrisch, M. Krstajic, T. Schreck, and D. A. Keim. The News Auditor: Visual Exploration of Clusters of Stories. In K. Matkovic and G. Santucci, editors, EuroVA 2012: International Workshop on Visual Analytics. The Eurographics Association, 2012.

[8] K. Bowers. Personal communication, November 4, 2016

[9] A. J. Bradley, H. Mehta, M. Hancock, and C. Collins. Visualization, Digital Humanities, and the Problem of Instrumentalism. In Workshop on Visualization for the Digital Humanities, IEEE VIS 2016, Baltimore, Maryland, USA, October 24th, 2016.

[10] M. Brehmer, S. Ingram, J. Stray, and T. Munzner. Overview: The Design, Adoption, and Analysis of a Visual Document Mining Tool for Investigative Journalists. Visualization and Computer Graphics, IEEE Transactions on, 20(12):2271-2280, Dec 2014.

[11] L. Byron and M. Wattenberg. Stacked Graphs - Geometry \& Aesthetics. Visualization and Computer Graphics, IEEE Transactions on, 14(6):1245-1252, Nov 2008.

[12] M. F. Cheema, S. Jänicke, and G. Scheuermann. AnnotateVis: Combining Traditional Close Reading with Visual Text Analysis. In Workshop on Visualization for the Digital Humanities, IEEE VIS 2016, Baltimore, Maryland, USA, October 24th, 2016.

[13] P. Christen. A Comparison of Personal Name Matching: Techniques and Practical Issues. In Data Mining Workshops, 2006. ICDM Workshops 2006. Sixth IEEE International Conference on, pages 290-294. IEEE, 2006.

[14] C. Collins, S. Carpendale, and G. Penn. Docuburst: Visualizing document content using language structure. In Computer Graphics Forum volume 28, pages 1039-1046. Wiley Online Library, 2009.

[15] C. Collins, F. Viegas, and M. Wattenberg. Parallel Tag Clouds to explore and analyze faceted text corpora. In Visual Analytics Science and Technology, 2009. VAST 2009. IEEE Symposium on, pages 91-98, Oct 2009.

[16] M. Correll, M. Witmore, and M. Gleicher. Exploring collections of tagged text for literary scholarship. Computer Graphics Forum, 30(3):731-740, 2011.

[17] J. J. Duggan. A Concordance of the Chanson de Roland. Ohio State University Press, 1969.

[18] M. El-Assady, V. Gold, W. Jentner, M. Butt, K. Holzinger, and D. A Keim. VisArgue - A Visual Text Analytics Framework for the Study of Deliberative Communication. In Proceedings of The International Conference on the Advances in Computational Analysis of Political Text (PolText2016), pages 31-36. University of Zagreb, 2016.

[19] S. Gaunt and S. Kay. The Cambridge Companion to Medieval French Literature. Cambridge Companions to Literature. Cambridge University Press, 2008

[20] Z. Geng, T. Cheesman, R. S. Laramee, K. Flanagan, and S. Thiel ShakerVis: Visual analysis of segment variation of German translations of Shakespeare's Othello. Information Visualization, 2013.
[21] F. Gibbs and T. Owens. Building Better Digital Humanities Tools: Toward broader audiences and user-centered designs. Digital Humanities Quarterly, 6(2), 2012.

[22] P. Goffin, W. Willett, J.-D. Fekete, and P. Isenberg. Exploring the Placement and Design of Word-Scale Visualizations. Visualization and Computer Graphics, IEEE Transactions on, 20(12):2291-2300, Dec 2014.

[23] J. M. Green. 'On Pe is nis bute chatering': cyberpragmatics and the paratextual anatomy of Twitter. Studies in Variation, Contacts and Change in English, 15, 2014.

[24] M. Harrower and C. A. Brewer. ColorBrewer.org: An Online Tool for Selecting Colour Schemes for Maps. The Cartographic Journal, 40(1):27-37, 2003.

[25] F. Heimerl, S. Koch, H. Bosch, and T. Ertl. Visual Classifier Training for Text Document Retrieval. Visualization and Computer Graphics, IEEE Transactions on, 18(12):2839-2848, Dec 2012.

[26] B. Heuwing, T. Mandl, and C. Womser-Hacker. Methods for UserCentered Design and Evaluation of Text Analysis Tools in a Digital History Project. Proceedings of the Association for Information Science and Technology, 53(1):1-10, 2016.

[27] S. Howell, M. Kelleher, A. Teehan, and J. Keating. A Digital Humanities Approach to Narrative Voice in The Secret Scripture: Proposing a New Research Method. Digital Humanities Quarterly, 8(2), 2014.

[28] S. Jänicke. Valuable Research for Visualization and Digital Humanities: A Balancing Act. In Workshop on Visualization for the Digital Humanities, IEEE VIS 2016, Baltimore, Maryland, USA, October 24th, 2016.

[29] S. Jänicke, T. Efer, M. Büchler, and G. Scheuermann. Designing Close and Distant Reading Visualizations for Text Re-use. In International Conference on Computer Vision, Imaging and Computer Graphics, pages 153-171. Springer International Publishing, 2014.

[30] S. Jänicke, J. Focht, and G. Scheuermann. Interactive Visual Profiling of Musicians. Visualization and Computer Graphics, IEEE Transactions on, 22(1):200-209, Jan 2016.

[31] S. Jänicke, G. Franzini, M. F. Cheema, and G. Scheuermann. On Close and Distant Reading in Digital Humanities: A Survey and Future Challenges. In R. Borgo, F. Ganovelli, and I. Viola, editors, Eurographics Conference on Visualization (EuroVis) - STARs. The Eurographics Association, 2015

[32] S. Jänicke, G. Franzini, M. F. Cheema, and G. Scheuermann. Visual Text Analysis in Digital Humanities. Computer Graphics Forum, 2016.

[33] S. Jänicke and A. Gessner. A Distant Reading Visualization for Variant Graphs. In Proceedings of the Digital Humanities 2015, 2015.

[34] S. Jänicke, A. Gessner, M. Büchler, and G. Scheuermann. Visualizations for Text Re-use. GRAPP/IVAPP, pages 59-70, 2014.

[35] S. Jänicke, A. Geßner, G. Franzini, M. Terras, S. Mahony, and G. Scheuermann. TRAViz: A Visualization for Variant Graphs. Digital Scholarship in the Humanities, 30(suppl 1):i83-i99, 2015.

[36] S. Jänicke and D. Wrisley. Visualizing Mouvance: Towards a Visual Analysis of Variant Medieval Text Traditions. Digital Scholarship in the Humanities (to appear), 2017.

[37] S. Jänicke and D. J. Wrisley. Visualizing Mouvance: Towards an Alignment of Medieval Vernacular Text Traditions. In Proceedings of the Digital Humanities 2016, 2016.

[38] M. L. Jockers. Macroanalysis: Digital Methods \& Literary History. University of Illinois Press, 2013.

[39] D. Keim and D. Oelke. Literature Fingerprinting: A New Method for Visual Literary Analysis. In Visual Analytics Science and Technology, 2007. VAST 2007. IEEE Symposium on, pages 115-122, Oct 2007.

[40] D. A. Keim, F. Mansmann, J. Schneidewind, J. Thomas, and H. Ziegler. Visual Analytics: Scope and Challenges. In Visual Data Mining, pages 76-90. Springer, 2008.

[41] E. J. Kenney. Textual Criticism, 2012. https://www.britannica. com/topic/textual-criticism (Retrieved 2017-03-06).

[42] S. Koch, H. Bosch, M. Giereth, and T. Ertl. Iterative Integration of Visual Insights during Scalable Patent Search and Analysis. IEEE Transactions on Visualization and Computer Graphics, 17(5):557-569, May 2011.

[43] S. Koch, M. John, M. Worner, A. Muller, and T. Ertl. VarifocalReader In-Depth Visual Analysis of Large Text Documents. Visualization and 
Computer Graphics, IEEE Transactions on, 20(12):1723-1732, Dec 2014.

[44] K. Kucher and A. Kerren. Text Visualization Techniques: Taxonomy, Visual Survey, and Community Insights. In 2015 IEEE Pacific Visualization Symposium (PacificVis), pages 117-121, April 2015.

[45] N. McCurdy, J. Lein, K. Coles, and M. Meyer. Poemage: Visualizing the Sonic Topology of a Poem. Visualization and Computer Graphics, IEEE Transactions on, 22(1):439-448, Jan 2016.

[46] T. Munzner. A nested model for visualization design and validation. Visualization and Computer Graphics, IEEE Transactions on, 15(6):921928, 2009.

[47] T. Munzner. Visualization Analysis \& Design. CRC Press, 2014.

[48] S. B. Needleman and C. D. Wunsch. A general method applicable to the search for similarities in the amino acid sequence of two proteins. Journal of molecular biology, 48(3):443-453, 1970.

[49] D. Oelke, D. Kokkinakis, and D. A. Keim. Fingerprint Matrices: Uncovering the dynamics of social networks in prose literature. In Computer Graphics Forum, volume 32, pages 371-380. Wiley Online Library, 2013.

[50] P. Riehmann, H. Gruendl, M. Potthast, M. Trenkmann, B. Stein, and B. Froehlich. WORDGRAPH: Keyword-in-Context Visualization for NETSPEAK's Wildcard Search. Visualization and Computer Graphics, IEEE Transactions on, 18(9):1411-1423, Sept 2012.

[51] P. Riehmann, M. Potthast, B. Stein, and B. Froehlich. Visual Assessment of Alleged Plagiarism Cases. Computer Graphics Forum, 34(3):61-70, 2015.

[52] G. Roe. Intertextuality and Influence in the Age of Enlightenment: Sequence Alignment Applications for Humanities Research. In Proceedings of the Digital Humanities 2012, 2012.

[53] B. Schmidt. Zooming In and Out: Close and Distant Reading., 2013. http://benschmidt.org/dighist $13 / ? \mathrm{p}=120$ (Retrieved 2017-06-27)

[54] B. Shneiderman. The Eyes Have It: A Task by Data Type Taxonomy for Information Visualizations. In Visual Languages, Proceedings, pages 336-343, 1996.

[55] H. Siirtola, T. Säily, T. Nevalainen, and K.-J. Räihä. Text Variation Explorer: Towards interactive visualization tools for corpus linguistics. International Journal of Corpus Linguistics, 19(3):417-429, 2014.

[56] S. Silvia, R. Etemadpour, J. Abbas, S. Huskey, and C. Weaver. Visualizing Variation in Classical Text with Force Directed Storylines. In Workshop on Visualization for the Digital Humanities, IEEE VIS 2016, Baltimore, Maryland, USA, October 24th, 2016.

[57] T. F. Smith and M. S. Waterman. Identification of common molecular subsequences. Journal of molecular biology, 147(1):195-197, 1981.

[58] H. Strobelt, D. Oelke, B. C. Kwon, T. Schreck, and H. Pfister. Guidelines for Effective Usage of Text Highlighting Techniques. Visualization and Computer Graphics, IEEE Transactions on, 22(1):489-498, Jan 2016.

[59] R. Vuillemot, T. Clement, C. Plaisant, and A. Kumar. What's being said near "Martha"? Exploring name entities in literary text collections. In Visual Analytics Science and Technology, 2009. VAST 2009. IEEE Symposium on, pages 107-114, Oct 2009.

[60] B. Walsh, C. Maiers, G. Nally, J. Boggs, and P. P. Team. Crowdsourcing individual interpretations: Between microtasking and macrotasking. Literary and Linguistic Computing, 29(3):379-386, 2014.

[61] M. Wattenberg and F. Viegas. The Word Tree, an Interactive Visual Concordance. Visualization and Computer Graphics, IEEE Transactions on, 14(6): 1221-1228, Nov 2008

[62] D. Wheeles and K. Jensen. Juxta Commons. In Proceedings of the Digital Humanities 2013, 2013.

[63] P. Zumthor. Toward a Medieval Poetics. University of Minnesota Press, 1992. 